\title{
Study protocol for improving asthma outcomes through cross-cultural communication training for physicians: a randomized trial of physician training
}

Minal R Patel ${ }^{1,2^{*}}$, Lara J Thomas ${ }^{1}$, Kausar Hafeez ${ }^{1}$, Matthew Shankin ${ }^{1}$, Margaret Wilkin ${ }^{1}$ and Randall W Brown ${ }^{1,2}$

\begin{abstract}
Background: Massive resources are expended every year on cross-cultural communication training for physicians. Such training is a focus of continuing medical education nationwide and is part of the curriculum of virtually every medical school in America. There is a pressing need for evidence regarding the effects on patients of cross-cultural communication training for physicians. There is a need to understand the added benefit of such training compared to more general communication. We know of no rigorous study that has assessed whether cross-cultural communication training for physicians results in better health outcomes for their patients. The current study aims to answer this question by enhancing the Physician Asthma Care Education (PACE) program to cross cultural communication (PACE Plus), and comparing the effect of the enhanced program to PACE on the health outcomes of African American and Latino/Hispanic children with asthma.
\end{abstract}

Methods/Design: A three-arm randomized control trial is used to compare PACE Plus, PACE, and usual care. Both PACE and PACE Plus are delivered in two, two-hour sessions over a period of two weeks to 5-10 primary care physicians who treat African American and Latino/Hispanic children with asthma. One hundred twelve physicians and 1060 of their pediatric patients were recruited who self-identify as African American or Latino/Hispanic and experience persistent asthma. Physicians were randomized into receiving either the PACE Plus or PACE intervention or into the control group. The comparative effectiveness of PACE and PACE Plus on clinician's therapeutic and communication practices with the family/patient, children's urgent care use for asthma, asthma control, and quality of life, and parent/caretaker satisfaction with physician performance will be assessed. Data are collected via telephone survey and medical record review at baseline, 9 months following the intervention, and 21 months following the intervention.

Discussion: This study aims to reduce disparities in asthma outcomes among African American and Latino/Hispanic children through cross-cultural communication training of their physicians and assessing the added value of this training compared to general communication. The results of this study will provide important information about the value of cross-cultural training in helping to address persistent racial disparities in outcomes.

Trial registration: ClinicalTrials.gov: NCT01251523 December 1, 2010

Keywords: Asthma, Randomized controlled trials, Physicians, Communication, Health disparities

\footnotetext{
* Correspondence: minalrp@umich.edu

'Center for Managing Chronic Disease, 1415 Washington Heights, Ann Arbor, MI 48109-2029, USA

2Department of Health Behavior and Health Education, University of

Michigan School of Public Health, 1415 Washington Heights, Ann Arbor, MI 48109-2029, USA
}

\section{Biomed Central}

(c) 2014 Patel et al.; licensee BioMed Central Ltd. This is an Open Access article distributed under the terms of the Creative Commons Attribution License (http://creativecommons.org/licenses/by/4.0), which permits unrestricted use, distribution, and reproduction in any medium, provided the original work is properly credited. The Creative Commons Public Domain Dedication waiver (http://creativecommons.org/publicdomain/zero/1.0/) applies to the data made available in this article, unless otherwise stated. 


\section{Background}

Asthma affects 7.1 million U.S. children and is the main reason children use urgent care services for a chronic disease [1]. In 2009, 1 in 5 children with asthma sought care in an emergency department [2]. Asthma prevalence among children and adolescents is estimated to be 9.6\% overall, and is highest among non-Hispanic black children (17.0\%) [2]. Hispanic/Latino and non-Hispanic black children have greater asthma-related risk exposures than non-Hispanic Whites, and are at higher risk for emergency department visits, hospitalizations, and death from asthma [3-6]. They exhibit lower adherence to medication and only about one-third report using long-term controller medicine or receiving asthma care plans from their clinicians [2]. Poor outcomes are especially striking among African American children, who have a $260 \%$ higher emergency department (ED) visit rate, a $250 \%$ higher hospitalization rate, and a 500\% higher death rate from asthma than their White counterparts [7]. Because the risk, severity, and control of asthma are all influenced by a combination of genetic, social, and environmental factors, reducing the impact of the condition has proven difficult. However, enhancing clinical care in populations with marked disparities is regarded as a priority effort $[8,9]$. Asthma is most often managed in the primary care setting and $80 \%$ of those affected never visit an asthma specialist [2]. Given the high prevalence of asthma in children and the associated frequency of visits to primary care practitioners, these health care providers are the asthma patients' primary source of care and education. Thus improving asthma clinical care in primary care providers offers the potential to reduce the burden of asthma in underserved populations.

The uncertainty of physicians in their interactions with minority patients, the clinicians' beliefs or stereotypes regarding the health behavior of minority patients, and patient response to perceived negative experiences with providers all have been discussed extensively in the literature [10]. The dramatic demographic shifts occurring in the United States mean that increasing numbers of minority patients are being seen in the health care system and, for the foreseeable future, the majority of the physicians who treat them are unlikely to be of their same racial or ethnic background. Further, it is increasingly expected that health care institutions account for the social and cultural diversity of the U.S. population [11] especially as it relates to clinical practice, and performance expectations for practicing physicians are also very high.

Massive resources are expended every year on crosscultural communication training for physicians. Such training is a focus of continuing medical education nationwide and is part of the curriculum of virtually every medical school in America. There is a pressing need for evidence regarding the effects on patients of crosscultural communication training for physicians. There is a need to understand the added benefit of such training compared to training in more general communication and patient-physician partnership skills [12]. However, we know of no rigorous study that has assessed whether cross-cultural communication training for physicians results in better health outcomes for their patients.

\section{Adapting the Physician Asthma Care Education Program}

The Physician Asthma Care Education (PACE) Program is a two-part interactive, multi-media educational seminar developed two-decades ago and widely disseminated to improve physician awareness, ability, and use of communication and therapeutic techniques for reducing the effects of asthma on children and their families. Efficacy and effectiveness trials have shown that patients of PACE-trained physicians demonstrate improvements in long-term outcomes including fewer symptoms and less health care use $[13,14]$. Importantly, no more time is required in the patient-physician encounter by PACEtrained physicians. This general communication intervention provides an effective comparison to investigate the added value of training in cross-cultural communication. PACE was enhanced to focus on cross-cultural communication training. This enhanced intervention, PACE Plus, was developed with cultural anthropology experts and piloted on three occasions with 31 primary care physicians. Unpublished data reveal that the majority of participants believed the training to be very useful and relevant, and participants demonstrated significant improvement in their understanding of cross-cultural communication, and increased confidence in engaging in tailored communication with underserved populations.

\section{Methods/Design}

This study used a randomized controlled design with three arms: control, the original PACE intervention, and PACE Plus intervention (enhanced with cross-cultural communication training) to test the comparative effectiveness of PACE with cross-cultural communication training, a proven asthma-based communication intervention for physicians. All study procedures were reviewed and approved by the University of Michigan Health Sciences and Behavioral Sciences Institutional Review Board (IRB-HSBS HUM\#00030087).

\section{Study hypotheses}

The study addressed two questions: 1) Does cross-cultural communication training (PACE Plus) produce better outcomes for African American and Latino/Hispanic children with asthma, and their respective parents, than a general communication training program (PACE)?; 2) Compared 
to the control group, is PACE, already shown to be effective with the general population of patients, effective when used specifically with African American and Latino/ Hispanic children with asthma, and their parents?

The study hypothesis is that there will be positive outcomes for patients of physicians in both interventions but better outcomes for those patients whose doctors participate in the cross-cultural communication training (PACE Plus). That is, PACE Plus compared to PACE will result in reductions in children's health care use for asthma, improved symptom experience for the children, greater parent/caretaker satisfaction with the physician's performance, enhanced asthma related quality of life for parents/caretakers of the patients, higher levels of confidence and value placed by physicians on skills needed when working cross culturally, and increased use of National Asthma Education and Prevention Program (NAEPP) recommended therapies by physicians. It is also hypothesized that PACE compared to a control group will produce better outcomes on the aforementioned, six dimensions.

\section{Sample size determination}

The sample size of 1002 children and their parents total at baseline was determined by power calculations using the primary outcome of proportion of subjects with emergency department (ED) visits in the last twelve months taking into account a $25 \%$ attrition rate over 24 months leaving 801 total subjects at the end of the study. The sample size calculation is based on the assumption that the clustering of patients within physician practices is negligible, which is justified from the previous PACE study [14]. In that prior research, the intracluster correlation values were close to zero ( 0.24 at the physician level; 0.003 at the site level). In analysis, such correlation will be adjusted for, if necessary. In the previous PACE study [14], the baseline proportion of patient participants with an ED visit was $30 \%$ and this was decreased by $15 \%$ in 24 months. For the current study, there will be $80 \%$ power to detect a more conservative difference of $12 \%$ or more in the proportion of patient participants with an ED visit in 24 months at the significance level of 0.05 .

In the previous PACE study, those in the intervention group decreased by a little more than one mean emergency office visit and 0.09 mean hospitalizations [14]. With the sample size in the current study, there will be statistical power to detect changes at that level for hospitalization and at much lower levels than in the previous PACE study for emergency office visits. Given the relatively rare occurrence of emergency department visits, the sample size needed to detect changes is relatively large; this gives an advantage in having sufficient statistical power to detect small changes in other outcomes, including symptoms, patient satisfaction and asthma related quality of life.

\section{Physician and parent recruitment Physicians}

A total of 112 physicians were recruited in Atlanta, GA, the Bronx, NY and several communities in Michigan. To be eligible, physicians met the inclusion criteria outlined in Table 1. The sites chosen represented areas with a large African American and Hispanic/Latino populations being served by physicians that did not self-identify as African American and Hispanic/Latino.

To recruit physicians, a roster of potential physician participants was generated using membership lists from, e.g., local professional societies, asthma coalitions, and yellow-pages listings. Recruitment packets were initially mailed to potential candidates. The packet included a cover letter from the local physician co-investigator, a brochure describing the study, a brief screening form and questionnaire with a self-addressed, stamped envelope. Seven to ten days after the mailing, the recruitment

Table 1 Inclusion criteria for participation in current RCT

\begin{tabular}{|c|c|c|}
\hline Physician criteria & Patient criteria & Parent criteria \\
\hline $\begin{array}{l}\text { 1. Licensed physician in practice - board certified } \\
\text { pediatrics/family medicine }\end{array}$ & $\begin{array}{l}\text { 1. Treated by the participating physician during the } \\
\text { study intake period }\end{array}$ & $\begin{array}{l}\text { 1. Has primary responsibility for the } \\
\text { child's care }\end{array}$ \\
\hline 2. Treating children with asthma & 2. Age $1-16$ years & $\begin{array}{l}\text { 2. Self-identified as African American } \\
\text { or Hispanic/Latino }\end{array}$ \\
\hline 3. Full-time (>25 hrs.) in a practice in GA, MI or NY & $\begin{array}{l}\text { 3. Diagnosis of asthma by a physician using NAEPP } \\
\text { Guidelines (alternative descriptions such as reactive } \\
\text { airway disease, bronchitis, or wheezy bronchitis were } \\
\text { not accepted) }\end{array}$ & 3. Has access to a telephone \\
\hline $\begin{array}{l}\text { 4. Not Hispanic/Latino or African American in ethnicity/ } \\
\text { race (this allowed us to test the PACE Plus components } \\
\text { that addressed cross cultural communication) }\end{array}$ & $\begin{array}{l}\text { 4. Has persistent asthma as defined by NAEPP } \\
\text { classification for asthma severity }\end{array}$ & 4. Consents to participate \\
\hline 5. Consented to participate & $\begin{array}{l}\text { 5. Does not have other chronic disorders that cause } \\
\text { pulmonary complications, e.g. sickle cell disease }\end{array}$ & \\
\hline $\begin{array}{l}\text { 6. Willing to generate a roster of pediatric African } \\
\text { American and Hispanic/Latino asthma patients }\end{array}$ & 6. Self-identify as African American or Hispanic/Latino & \\
\hline
\end{tabular}


coordinator called each physician office, spoke to a practice manager and arranged a day and time for the physician co-investigator to speak directly with the potential physician recruit. Personal contact by a local clinical practice leader was an important strategy for recruiting physicians [15-17]. Multiple visits and phone calls to physician offices and practice managers were not uncommon due to busy clinic schedules.

Physicians were told that participation involved generating a roster of their patients with asthma, completing 3 self-administered interviews over 2 years, and willingness to be randomly assigned to one of three study conditions. Every effort was made to minimize physician or office staff burden. For example, research-related meetings with office staff were kept to a minimum. All enrolled physicians received, a $\$ 50$ gift card for completing each of the three data collection questionnaires and $\$ 50$ for their office for providing a patient list in Year 1 and health care use data at the end of the study. Physicians randomized to either the PACE or PACE Plus interventions arms received $5 \mathrm{CME}$ credits for completing the intervention sessions.

\section{Child and parent recruitment}

A total of 1,060 patients were recruited from the pool of patients provided by enrolled physicians. To be eligible, the child needed to meet the inclusion criteria listed in Table 1 and their parent or caregiver needed to meet the eligibility criteria as well.

Initially, the office staff of a participating physician was asked to provide a list of patients who met the eligibility criteria using office records. The patient list was provided to research staff. Eligible patients/caretakers were then mailed a recruitment packet containing a cover letter from the participating physician inviting them to enroll, a study overview page, two copies of the consent form, a health information screener form, a decline postcard, and a stamped self-addressed envelope for returning a copy of the consent and screener form.

Following the mailed information, patients received recruitment phone calls, which provided information about the study's purpose, design, randomization, and data collection. Calls were made by trained bilingual staff, to ensure the same information was provided to all potential participants. Participants could opt to complete the consent and screener via paper and mail them back, via telephone with a trained staff member. Once consent was received by the research staff, parents were contacted by a telephone interviewer and scheduled for a baseline interview. The recruitment scripts, consent forms, and the telephone interviews were available in both English and Spanish. Children/parents subsequently followed physicians into one of the three study arms as randomized.
Parents were not informed if their physician was participating in the intervention.

The following ongoing strategies were employed to enhance recruitment and retention. 1) caretakers received a \$30 gift card following completion of the baseline data-collection interview and a $\$ 20$ gift card for each subsequent completed follow-up interview; 2) Mother's and Father's Day cards were mailed annually to maintain a personal connection; 3) a study newsletter, mailed biannually, provided updates on study progress and basic asthma information; 4) repeated attempts were made over a year to check for reconnected phone lines; 5) the study team maintained contact with physician offices and periodically asked for updated contact information for enrolled participants.

\section{Randomization}

\section{Experimental design and randomization}

A randomized controlled design was used in this study. At the beginning of the study, a permuted block randomization schedule was created using the RAN TBL and RANUNI functions in SAS to generate blocks of two for random assignment of physicians. Data are collected from three sites, Atlanta, Georgia, New York City, New York, and cities throughout Michigan. It is recognized that geographic site as well as size of practice of the physicians selected for study could impact on results. Thus, randomization was stratified by geographic area (three levels, Atlanta area, metropolitan New York, and Michigan) and size of practice (two levels, solo practice and multiple-physician practices). This randomization ensured that each type of practice and geographic area would be evenly distributed across the three arms of the study, which reduces bias due to these potentially confounding factors in the comparative analysis. Once physicians consented to participate, they were placed into one of the three arms of the study according to the randomization schedule with 44 physicians assigned to PACE, 36 to PACE PLUS and 32 to control (see sample size determination) with a total of 112 physicians and 1062 patients following those physicians into randomization.

Because this evaluation is being conducted for the purpose of recommending interventions for adoption, considerations about external validity of the results are important. One strength of this sample is that it includes physicians from practices in three large metropolitan areas. Thus, although the physician sample involved is not drawn from the entire U.S. population of clinicians, the study results will be somewhat representative of a wide variety of practice sites. Further, because background and practice characteristics of participating physicians will be assessed, it will be possible to make comparisons to the broader group of clinicians practicing in the U.S., as well 
as judgments about the extent to which the interventions are influenced by these characteristics.

Another threat to external validity, multiple-treatment interference, will be addressed by questionnaire items that will allow us to measure whether physicians have been involved in any other professional education efforts focusing on asthma care and education and cross-cultural communication training.

\section{Theory and content of interventions}

Two interventions were compared in this study: PACE, and a culturally enhanced version of PACE: PACE Plus. PACE is based on the Model for Managing Chronic Disease (MMCD) [18]. The theoretical foundation for the MMCD includes principles of self-regulated learning and constructs from social cognitive theory [19-21]. Self-regulatory processes have been shown to have an impact on ability to organize, prioritize, and correct behavior [20]. Social cognitive theory is a particularly appealing basis for these interventions as they address management of a chronic disease, that is, a condition the physician can expect to treat over a long period of time. The theory posits that personal, social, and behavioral influences interact to cause behavior. In the applications of the theory, the physician self-regulates his/her own behavior to better achieve desired responses from the patients: more effective at-home management of the child's condition, greater adherence to the physician's recommendations by the family, and greater satisfaction with the physician's care. The theory and previous work with PACE $[13,14,22,23]$ supports the idea that influence becomes reciprocal. The physician is reinforced by the patient's positive response and is thus further inclined to collaborate with the patient, communicate more effectively, and improve asthma care. The patient's behavior is similarly reinforced through satisfaction with the care and results. Theoretically, this cyclical reinforcement over time should lead to stronger and stronger partnerships between family and physician in managing the child's illness.

\section{PACE}

PACE is targeted to primary care physicians. The MMCD framework informs the intervention components. Physicians make decisions and take action based on previous experience and the consequences they anticipate. When desired consequences are achieved, behavior is reinforced and motivation to use the behavior again increases. This motivation is experienced as an increased sense of selfefficacy or confidence that the behavior can be successfully used again to achieve similar or better results. Learning is enhanced by self-regulation, that is, the learner's efforts to observe, evaluate, and react to his or her own responses to a problem. A primary channel for learning is observing and drawing conclusions in vicarious situations. Physicians can learn from role models and/or other physicians who have excellent treatment and counseling skills.

PACE consists of two, 2-hour sessions offered to groups of $4-6$ primary care physicians two weeks apart in an interactive group format. The content of PACE focuses on enhancing communication, therapeutic practice, and the ability of physicians to foster effective management of asthma in their pediatric patients. Since the step-care approach to asthma based on the National Asthma Education Prevention Program (NAEPP) Expert Guidelines for the Diagnosis and Management of Asthma is likely to require a few office visits [8], communication can be accomplished within the framework of these visits. Physicians are therefore provided with an approach in this intervention that can be easily introduced into their ongoing practices, requiring no increases in time and having maximum impact on the patient's ability to retain and use treatment advice and education.

Table 2 provides an overview of program content for PACE and PACE Plus. Both interventions enable the physician to use a process of self-regulation to improve his/ her treatment decisions and counseling via mini-lectures, case studies, videos, and interactive discussion. In PACE, the first segment of the intervention is designed to help physicians to improve their clinical management of asthma through NAEPP protocols for effective treatment [8]. The second segment of the intervention provides the physicians with messages describing strategies to help families manage effectively. The third segment of the intervention teaches physicians techniques that will improve their ability to communicate advice to families, and the fourth segment focuses on up-to-date billing and coding practices for asthma management. The intervention is facilitated by a respected pediatric pulmonologist, primary care physician, and behavioral scientist.

\section{PACE plus}

PACE Plus is tailored to cross-cultural communication with a specific focus on working with African American and Latino/Hispanic families. Although it is the same length and intensity as PACE, PACE Plus content differs from PACE in that the billing and coding component is replaced with a mini-lecture segment focused specifically on cross-cultural communication strategies and working effectively with a translator. The content addresses the range of perceptions and experiences of children with asthma and their parents that affect asthma management. This content, gleaned from the literature $[24,25]$ and the clinical experiences of the physician co-investigators, includes topics relevant to the target populations, such as patient perceptions, beliefs, fears, and preferences. Case studies in PACE Plus are embedded in the context of working with African American and Latino/Hispanic 
Table 2 Comparison of physician interventions

\begin{tabular}{lll}
\hline & PACE & PACE PLUS \\
\hline DELIVERY FORMAT: & $\begin{array}{l}\text { Seminar comprises 2 face-to-face group meetings } \\
\text { lasting } 2 \frac{1 / 2}{2} \text { hrs. each, held over a 2-week period. } \\
\text { Format is a combination of mini lecture, video, }\end{array}$ & $\begin{array}{l}\text { Original PACE delivery format with integrated cultural } \\
\text { competence skills and components. }\end{array}$ \\
case studies, \& discussion. & \\
FACILITATORS: & $\begin{array}{l}\text { Includes an asthma specialist, behavioral science } \\
\text { expert, and a primary care physician. }\end{array}$ & $\begin{array}{l}\text { Includes an asthma specialist, behavioral science expert } \\
\text { skilled in cross-cultural communication, and a primary } \\
\text { care physician. }\end{array}$
\end{tabular}

\section{CURRICULUM COMPONENTS}

CLINICAL ASPECTS OF ASTHMA AND LONG-TERM MANAGEMENT

COMMUNICATION STRATEGIES

PATIENT EDUCATION MESSAGES

CASES

ADDITIONAL COMPONENTS

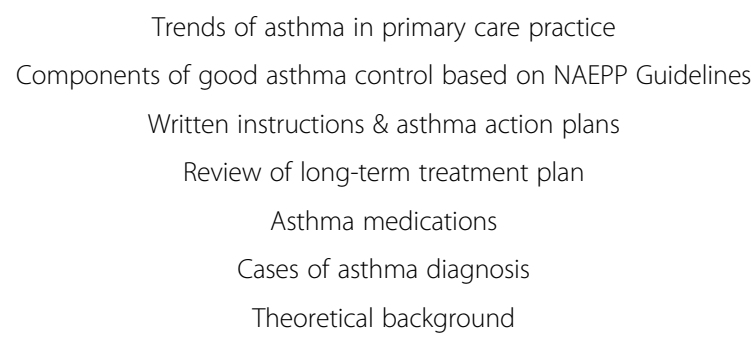

Evidence on asthma medication adherence and from efficacy trial of PACE

Video of communication strategies + discussion

Introduction of 10 communications strategies

Use of self-rating scale and communications strategies with patients between intervention sessions + reflection with group

Video of patient education and presentation of core asthma messages and discussion

Asthma treatment cases + discussion

Billing and coding asthma visits

-review of billing and coding practices for asthma education and counseling via mini-lecture and video. Segment is especially enhanced when facilitated by a billing and coding expert

\begin{abstract}
Additional discussion questions concerning diverse patient groups specifically African American and Hispanic/Latino pediatric patients and families responding differently to messages

Review of case studies highlighting perceptions of treating asthma among patients of African American and Hispanic/Latino race/ethnicity

Cross-cultural communication

Review of concerns of African American and Hispanic/ Latino patients

Review of cross cultural communication strategies Video highlights cultural issues

Emphasis on communication barrier being more evident with patients of a different race/ethnicity \& means to reduce barriers
\end{abstract}

patients. The behavioral scientist facilitator in PACE Plus is an individual particularly skilled in cross-cultural communication.

\section{Control condition}

The control condition consisted of usual care. Usual asthma care in the United States recognizes the model of practice as established by National Asthma Education Prevention Program [8]. Participating control physicians acknowledged use of guideline-based care and were unrestricted in their acquisition of updated asthma CME during the study.

\section{Outcomes and measures}

Data are collected from three sources in this study: 1) enrolled physicians complete self-administered surveys,
2) caretakers of patients complete telephone surveys, and 3) health care use data collected via the physician's office records. Physician and parent/caregiver surveys are completed at baseline, 9 months following the intervention, and 21 months following the intervention. All physician surveys are collected via Qualtrics, an online survey program, or on paper and then returned to the research staff. Following completion of the baseline survey, physicians were randomized and notified of their assignment with further instructions for those randomized to the PACE or PACE Plus arms. Data are collected from parents/caretakers via telephone interviews with trained research assistants. Data are then entered twice into Qualtrics to ensure accuracy.

The primary study outcome is children's health care use for asthma. This includes the number of asthma-related 
emergency department visits, overnight hospitalizations, and urgent office visits. These measures are collected via self-report during the telephone interviews, and verified using medical records from the physician offices.

Other outcomes of interest include the following:

Clinical practices of the clinician: Physician's selfreported clinical practices. These questions focus on time spent with patients of different duration of care and asthma severity, as well as communication and counseling behaviors. Practice of communication and counseling is measured using the Teaching and Communication Behavior scale [26] which address the frequency, perceived helpfulness, and confidence with which the physician performs different communication strategies.

Knowledge and use of NAEPP guidelines: Physician's knowledge and familiarity with the NAEPP guidelines for asthma management and diagnosis, and their use of NAEPP recommended therapies.

Cultural competency: Physician's use of recommended cultural competence behaviors as well as expectations about their effect and confidence to employ them.

Asthma symptoms: Collected from the parent using items based on NAEPP guidelines to measure the intensity and level of symptoms.

Asthma Control Test: The validated 5-item Asthma Control Test (ACT) and children's 7-item ACT measure the effect of asthma on daily functioning, shortness of breath, nighttime and daytime asthma symptoms, rescue medication use, and overall parent-rated asthma control [27].

\section{Parent/caretaker satisfaction with physician} performance: These items were previously developed by the authors [13] tapping into measure physician performance regarding communication and counseling, and specific facets of general satisfaction with care. Parents/caretaker's views of the physician's cultural competence is assessed using items based on crosscultural communication training provided in the PACE Plus training.

Quality of life: The 13-item Pediatric Asthma Caregiver's Quality of Life scale measures limitations, anxieties, and fears caregivers experience due to their child's asthma [28].

Data are also collected in the physician survey on a wide variety of socio-demographic information, including practice arrangements, professional activities and previous involvement in cross-cultural communication training. Socio-demographic information related to the parent and child was also collected including availability of health insurance.

\section{Analysis}

Analyses will involve both discrete (dichotomous, counts, ordinal) and continuous (measurable) variables. Analysis techniques utilized will be based on the type of variables, categorical or continuous. Initially, descriptive statistics for the characteristics of the sample for each group will be produced. Then $t$ tests/Wilcoxon rank test, chi-squared tests and Fisher's exact test will be used to detect potential differences on demographic and practice variables between three randomized groups. If differences are found, adjustments in post-test measures, via subgroup and covariance analyses, will be made. Adjusted comparisons, controlling for variables that relate to the outcome will be performed using multivariate regression techniques. Adjusting for variables that relate to the outcome will increase the efficiency of the estimates by reducing the residual error, and correct for bias due to association between drop outs and these variables.

Separate analyses of physicians' characteristics related to each of the communication and cultural competence skills will be conducted. Multiple logistic regression will be used for dichotomous variables in each analysis with group assignment as an independent variable. Changes in the physicians' use of specific NAEPP recommended therapies will be examined through logistic regression.

Analyses of the parent interview data will be conducted to assess outcomes that are related to four categories of variables: 1) changes in the child's health care use for asthma 2) changes in the child's symptom status 3) changes in parents' views of physician performance; and 4) increase in asthma-related quality of life for parents. For changes in parents' views of physician counseling, multiple logistic regression will be used for each analysis with group assignment as an independent variable. The role of patient age and gender, severity of illness, tobacco exposure (as this is associated with increased asthma symptoms), patient insurance (private, Medicaid, or self-pay status), and other relevant influences will be identified for appropriate inclusion in models.

\section{Discussion}

Improving outcomes of underserved groups that are particularly vulnerable to difficulties with self-management and clinical care that may not be tailored to their values and preferences is a practice priority. This study aims to reduce disparities in asthma outcomes among African American and Latino/Hispanic children through cross-cultural 
communication training of their physicians and assessing the added value of this training compared to general communication. The results of this study will provide important information about the value of cross-cultural communication training in helping to address persistent racial disparities in asthma outcomes.

\section{Abbreviations}

PACE: Physician asthma care education; UM: University of Michigan; ED: Emergency department; PACE Plus: Physician asthma care education with cross cultural communication; NAEPP: National asthma education and prevention program; MMCD: Model for managing chronic disease; ACT: Asthma control test.

\section{Competing interests}

Dr. Patel, Ms. Thomas, Ms. Hafeez, Mr. Shankin, and Ms. Wilkin declare that they have no competing interests. Dr. Brown serves on the board of directors of the National Asthma Education Certification Board. Dr. Brown is on advisory board and speaker panels for Astra-Zeneca and Teva Pharmaceuticals.

Dr. Brown has served on past speaker panels for Aerocrine and Merck \& Co.

\section{Authors' contributions}

$\mathrm{RB}$ is the Principal Investigator of the study. LJT, KH, MS are involved in project management and/or intervention delivery. MW was involved in conception of design and analysis and interpretation of the data. All authors participated in the design of the trial, intervention, and/or measures. MRP and LJT drafted the manuscript; all authors reviewed, edited, and approved the final manuscript.

\section{Acknowledgements}

This trial was funded by R01HL093386-01-A2 from the Division of Lung Diseases of the National Heart, Lung, and Blood Institute of the National Institutes of Health. The authors would like to thank Meagan Shallcross and Harim Yoo for their assistance with this manuscript. This article is written in the loving memory of Dr. Noreen M. Clark whose lifelong asthma work has changed the lives of patients and informed physician practice. We would also like to thank all the physicians and parents who participated in this trial.

Received: 7 May 2014 Accepted: 12 June 2014

Published: 16 June 2014

\section{References}

1. Bloom B, Cohen RA, Freeman G: Summary health statistics for U.S. children: National Health Interview Survey, 2010. Vital Health Stat 10 2011, 250:1-80.

2. Centers for Disease Control and Prevention (CDC): Vital signs: asthma prevalence, disease characteristics, and self-management education: United States, 2001-2009. MMWR Morb Mortal Wkly Rep 2011 60(17):547-552.

3. Williams DR, Sternthal $M$, Wright RJ: Social determinants: taking the social context of asthma seriously. Pediatrics 2009, 123(Suppl 3):S174-S184.

4. Akinbami L, Moorman JE, Garbe PL, Sondik EJ: Status of childhood asthma in the United States, 1980-2007. Pediatrics 2009, 123(Suppl 3):S131-S145.

5. Stewart KA, Higgins PC, McLaughlin CG, Williams TV, Granger E, Croghan TW: Differences in prevalence, treatment, and outcomes of asthma among a diverse population of children with equal access to care: findings from a study in the military health system. Arch Pediatr Adolesc Med 2010, 164(8):720-726.

6. Canino G, Koinis-Mitchell D, Ortega AN, McQuaid EL, Fritz GK, Alegria M: Asthma disparities in the prevalence, morbidity, and treatment of Latino children. Soc Sci Med 2006, 63(11):2926-2937.

7. Akinbami L: The state of childhood asthma, United States, 1980-2005. Adv Data 2006, 381:1-24.

8. National Heart, Lung, and Blood Institute: National asthma education and prevention program. In Expert panel report 3: guidelines for the diagnosis and management of asthma. Bethesda MD: 2007.

9. Hegner R: The asthma epidemic: prospects for controlling an escalating public health crisis. Washington, DC: National Health Policy Forum, The George Washington University; 2000.
10. Saha S, Arbelaez JJ, Cooper LA: Patient-physician relationships and racial disparities in the quality of health care. Am J Public Health 2003, 93(10):1713-1719.

11. Wright RJ, Subramanian SV: Advancing a multilevel framework for epidemiologic research on asthma disparities. Chest 2007, 132(Suppl 5):757S-7695.

12. Lum CK, Korenman SG: Cultural-sensitivity training in U.S. medical schools. Acad Med 1994, 69(3):239-241.

13. Clark NM, Gong M, Schork MA, Evans D, Roloff D, Hurwitz M, Maiman L, Mellins RB: Impact of education for physicians on patient outcomes. Pediatrics 1998, 101(5):831-836.

14. Cabana MD, Slish KK, Evans D, Mellins RB, Brown RW, Lin X, Kaciroti N, Clark NM: Impact of physician asthma care education on patient outcomes. Pediatrics 2006, 117(6):2149-2157.

15. Levinson W, Dull VT, Roter DL, Chaumeton N, Frankel RM: Recruiting physicians for office-based research. Med Care 1998, 36(6):934-937.

16. Asch S, Connor SE, Hamilton EG, Fox SA: Problems in recruiting community-based physicians for health services research. J Gen Intern Med 2000, 15(8):591-599.

17. Hudson SV, Harris-Haywood S, Stange KC, Orzano AJ, Crabtree BF: Recruiting minority primary care practices into practice-based research. Med Care 2006, 44(7):696-700.

18. Clark NM: The use of self-regulation interventions in managing chronic disease. In Applications of self-regulated learning across diverse disciplines: a tribute to Barry J. Zimmerman. Edited by Schunk DH, Usher EL. North Carolina: Information Age Publishing; 2013:417-443.

19. Bandura A: Health promotion by social cognitive means. Health Educ Behav 2004, 31(2):143-164.

20. Zimmerman BJ, Schunk DH: Self-regulated learning and academic achievement: theoretical perspectives. 2nd edition. Mahwah, NJ: Lawrence Erlbaum; 2001.

21. Clark NM, Zimmerman BJ: A social cognitive view of self-regulated learning about health. Health Educ Res 1990, 5(3):371-379.

22. Clark NM, Gong M, Schork MA, Kaciroti N, Evans D, Roloff D, Hurwitz M, Maiman LA, Mellins RB: Long-term effects of asthma education for physicians on patient satisfaction and use of health services. Eur Respir $J$ 2000, 16(1):15-21.

23. Clark NM, Cabana MD, Nan B, Gong ZM, Slish KK, Birk NA, Kaciroti N: The clinician-patient partnership paradigm: outcomes associated with physician communication behavior. Clin Pediatr (Phila) 2008, 47(1):49-57.

24. Tumiel-Berhalter L, Zayas LE: Lay experiences and concerns with asthma in an urban Hispanic community. J Natl Med Assoc 2006, 98(6):875-880.

25. Berg J, Anderson NL, Tichacek MJ, Tomizh AC, Rachelefsky G: One gets so afraid: Latino families and asthma management-an exploratory study. J Pediatr Health Care 2007, 21(6):361-371.

26. Clark NM, Gong M, Schork MA, Maiman LA, Evans D, Hurwitz ME, Roloff D, Mellins RB: A scale for assessing health care providers' teaching and communication behavior regarding asthma. Health Educ Behav 1997, 24(2):245-256.

27. Schatz M, Sorkness CA, Li JT, Marcus P, Murray JJ, Nathan RA, Kosinski M, Pedergraft TB, Jhingran P: Asthma Control Test: reliability, validity, and responsiveness in patients not previously followed by asthma specialists. J Allergy Clin Immunol 2006, 117(3):549-556.

28. Juniper EF, Guyatt GH, Feeny DH, Ferrie PJ, Griffith LE, Townsend M: Measuring quality of life in the parents of children with asthma. Qual Life Res 1996, 5(1):27-34.

\section{doi:10.1186/1472-6920-14-118}

Cite this article as: Patel et al:: Study protocol for improving asthma outcomes through cross-cultural communication training for physicians: a randomized trial of physician training. BMC Medical Education 2014 14:118. 